\title{
Low-frequency scattering from two-dimensional perfect conductors
}

\author{
Hansen, Thorkild; Yaghjian, A.D
}

Published in:

Antennas and Propagation Society International Symposium

Link to article, DOI:

10.1109/APS.1991.174965

Publication date:

1991

Document Version

Publisher's PDF, also known as Version of record

Link back to DTU Orbit

Citation (APA):

Hansen, T., \& Yaghjian, A. D. (1991). Low-frequency scattering from two-dimensional perfect conductors. In Antennas and Propagation Society International Symposium (Vol. Volume 2, pp. 798-801). IEEE. https://doi.org/10.1109/APS.1991.174965

\section{General rights}

Copyright and moral rights for the publications made accessible in the public portal are retained by the authors and/or other copyright owners and it is a condition of accessing publications that users recognise and abide by the legal requirements associated with these rights.

- Users may download and print one copy of any publication from the public portal for the purpose of private study or research.

- You may not further distribute the material or use it for any profit-making activity or commercial gain

- You may freely distribute the URL identifying the publication in the public portal

If you believe that this document breaches copyright please contact us providing details, and we will remove access to the work immediately and investigate your claim. 


\author{
Thorkild B. Hansen* and Arthur D. Yaghjian \\ Electromagnetics Inśtitute Rome Laboratory \\ Technical University of Denmark Hanscom AFB, MA 01731
}

We have obtained exact expressions for the leading terms in the low-frequency expansions of the far fields scattered from three different types of two-dimensional perfect conductors: the cylinder with finite cross section, the cylindrical bump on an infinite ground plane, and the cylindrical dent in an infinite ground plane [1]. By inserting the low-frequency expansions of the incident flelds and Green's functions into exact integral equations for the surface current, we obtain integral equations for the leading terms in the low-frequency expansions of the surface currents. The leading terms in the low-frequency expansions of the scattered fields are obtained from simple integrations of these low-frequency expansions for the currents.

For the cylinder with finlte cross section the low-frequency scattered fleld for TM polarization is independent of the shape of the cross section of the cylinder and of order $1 / 1 \mathrm{n} k d$ where $d$ is a characteristic dimension of the cylinder. This low-frequency result can not be obtained from a corresponding statics fleld problem.

For IE polarization the scattered fleld is of order $(\mathrm{kd})^{2}$ and it consists of a contribution from a magnetic dipole along the axis of the cylinder and an electric dipole in a direction normal to the axis of the cylinder. The magnetic dipole moment is found directly from the area of the cross section of the cylinder. The electric dipole moment is found by solving two electrostatic problems and performing an integration of these two electrostatic solutions around the cylinder. These electrostatic solutions are determined from simple integral equations and depend only on the shape of the cylinder.

For the two-dimensional bump on a ground plane the low-frequency diffracted field for TM polarization is of order $(\mathrm{kd})^{2}$, where $d$ is a characteristic dimension of the bump. The low-frequency fleld is that of a magnetic dipole in the direction normal to the axis of the cylinder and parallel to the ground plane.

For the TE polarization the low-frequency diffracted fleld is also of order $(k d)^{2}$. It consists of a contribution from a magnetic dipole in the cylinder direction and an electric dipole in the direction normal to both the axis of the cylinder and the ground plane. The TE magnetic dipole moment is found directly from the area of the cross section of the bump.

Both the TM magnetic dipole field and the TE electric dipole field are written as a constant times a known simple function. It is proven that, remarkably, this constant, which depends only on the shape of the bump. is the same for the two polarizations. This constant can be found by solving elther a magnetostatic or electrostatic field problem and performing an integration of these solutions over the bump. This means that the TM-TE low-frequency diffracted fields for an arbitrarily shaped bump are completely determined by calculating one constant.

U.S. Government work not protected by U.S. copyright 
The low-frequency results for the two-dimensional dent in a ground plane, even more remarkably, are qualitatively the same as the results for the bump. That is, the TM-TE low-frequency diffracted fields for an arbitrarlly shaped dent are completely determined by calculating one constant that depends only on the shape of the dent.

These results are all derived for the case where the incldent plane wave propagates in a direction normal to the axis of the cylinder. However, they can easily be extended to oblique incidence using the standard procedure [2] based on the facts that the scatterer is perfectly conducting and the obliquely reflected field can be obtained from the normally reflected field by the same procedure.

We include a summary of the equations for the low-frequency far flelds produced by TM and TE polarized plane waves incident normally on a cylinder with finite cross section, a two-dimensional bump on an infinite ground plane, and a two-dimensional dent in an infinite ground plane. With $e^{\text {jwt }}$ time dependence:

I. Cylinder of finite cross section

A. TM polarization $\left(\bar{E}^{1}=\hat{z} e^{j k\left(x \cos \phi^{1}+y \sin \phi^{1}\right)}\right)$

$$
E_{z}^{s}(\bar{r})=\frac{1}{\ln k d} e^{-j \frac{\pi}{4}} \sqrt{\frac{\pi}{2}} \frac{e^{-j k r}}{\sqrt{k r}}
$$

B. IE polarization $\left(\bar{H}^{1}=\hat{z} e^{j k\left(x \cos \phi^{1}+y \sin \phi^{j}\right)}\right)$

$$
\begin{gathered}
H_{z}^{s}(\bar{r})=-\left(k d^{\prime}\right)^{2} \frac{e^{-j \frac{\pi}{4}}}{2 \sqrt{2 \pi}} \frac{e^{-j k r}}{\sqrt{k r}} \frac{1}{d^{2}}\left[A_{s}+C_{1} \sin \phi \sin \phi^{1}\right. \\
\left.+C_{2} \cos \phi \cos \phi^{\prime}+C_{3} \sin \left(\phi+\phi^{\prime}\right)\right] \\
C_{1}=\int_{s} \frac{\sigma^{\circ x}\left(\bar{r}^{\prime}\right)}{\varepsilon} x^{\prime} d s^{\prime}, C_{2}=\int_{s} \frac{\sigma^{\circ y}\left(\overline{r^{\prime}}\right)}{\varepsilon} y^{\prime} d s^{\prime}, C_{3}=-\int_{s} \frac{\sigma^{\circ y}\left(\bar{r}^{\prime}\right)}{\varepsilon} x^{\prime} d s^{\prime}
\end{gathered}
$$

$A_{s}$ is the area of the cross section of the cylinder and $\frac{\sigma^{0 x}}{\varepsilon}$ and $\frac{\sigma^{\circ y}}{\varepsilon}$ are the solutions to the static integral equations

$$
\frac{\partial x}{\partial s}=-f_{s} \frac{\partial}{\partial s^{\prime}} G^{\circ}\left(\bar{r}, \bar{r}^{\prime}\right) \frac{\sigma^{o x}\left(\bar{r}^{\prime}\right)}{\varepsilon} d s^{\prime}, \bar{r} \varepsilon s
$$




$$
\frac{\partial y}{\partial s}=-f_{s} \frac{\partial}{\partial s^{\prime}} G^{0}\left(\bar{r}, \bar{r}^{\prime}\right) \frac{\sigma^{o y}\left(\bar{r}^{\prime}\right)}{c} d s^{\prime}, \bar{r} \varepsilon s
$$

These expressions for the narrow cylinder of finite cross section rigorously conf 1rm the previous results of Van Bladel [3].

II. Two-dimensional bump on an infinite ground plane

A. TM polarization $\left(\bar{E}^{1}=\hat{z} e^{j k\left(x \cos \phi^{1}+y \sin \phi^{\prime}\right)}\right.$ )

$$
E_{z}^{d}(\bar{r})=-(k d)^{2} e^{-j \frac{\pi}{4}} \sqrt{\frac{2}{\pi}} \frac{e^{-j k r}}{\sqrt{k r}} \sin \phi \sin \phi^{\prime} \frac{B_{0}}{d^{2}}
$$

B. TE polarization $\left(\bar{H}^{1}=\hat{z} e^{j k\left(x \cos \phi^{1}+y \sin \phi^{1}\right)}\right)$

$$
\begin{gathered}
H_{2}^{d}(\vec{r})=-(k d)^{2} e^{-j \frac{\pi}{4}} \sqrt{\frac{2}{\pi}} \frac{e^{-j k r}}{\sqrt{k r}} \frac{1}{d^{2}}\left[A_{B}-B_{0} \cos \phi \cos \phi^{\prime}\right] \\
B_{0}=\int_{B} K_{2}^{O B}\left(\bar{r}^{\prime}\right) y^{\prime} d s^{\prime}
\end{gathered}
$$

$A_{B}$ is the area of the cross section of the bump and $K_{2}^{0 B}$ is the solution to the static integral equation

$$
-y=f_{\mathrm{BUB}_{1}} K_{2}^{0 D}\left(\bar{r}^{\prime}\right) G^{0}\left(\bar{r}, \bar{r}^{\prime}\right) d s^{\prime}, \bar{r} \approx B U B_{1}
$$

III. Two-dimensional dent in an infinite ground plane

A. TM polarization $\left(\bar{E}^{1}=\hat{z} e^{j k\left(x \cos \phi^{1}+y \sin \phi^{1}\right)}\right.$,

$$
E_{z}^{d}(\bar{r})=-(k d)^{2} e^{-j \frac{\pi}{4}} \sqrt{\frac{2}{\pi}} \frac{e^{-j k r}}{\sqrt{k r}} \sin \phi \sin \phi^{2} \frac{D_{0}}{d^{2}}
$$

B. TE polarization $\left(\bar{H}^{1}=\hat{z} e^{j k\left(x \cos \phi^{1}+y \sin \phi^{1}\right)}\right)$

$$
H_{z}^{d}(\bar{r})=(k d)^{2} e^{-j \frac{\pi}{4}} \sqrt{\frac{2}{\pi}} \frac{e^{-j k r}}{\sqrt{k r}} \frac{1}{d^{2}}\left[A_{D}+D_{0} \cos \phi \cos \phi^{1}\right]
$$




$$
D_{0}=\int_{D} K_{z}^{O D}\left(\bar{r}^{\prime}\right) y^{\prime} d s^{\prime}
$$

$A_{D}$ is the area of the cross section of the dent and $K_{z}^{O D}$ is the solution to the coupled static integral equations

$$
\int_{D} \frac{\partial}{\partial y^{\prime}} G^{0}\left(\bar{r}, \bar{r}^{\prime}\right) K_{z}^{O D}\left(\bar{r}^{\prime}\right) d s^{\prime}=\frac{1}{2}-H_{x}^{O D}(\bar{r}), \bar{r} \in A
$$

and

$$
f_{D} G_{x}^{0}\left(\bar{r}, \bar{r}^{\prime}\right) K_{z}^{0 D}\left(\bar{r}^{\prime}\right) d s^{\prime}+2 \int_{A} G^{0}\left(\bar{r}, \bar{r}^{\prime}\right) H_{x}^{0 D}\left(\bar{r}^{\prime}\right) d s^{\prime}=0, \bar{r} \varepsilon D
$$

The expressions (1), (2), (5), (6), (9), and (10) are used in the following companion paper to find the incremental length diffraction coefficients for narrow perfectly conducting cylinders, ridges on ground planes, and channels in ground planes.

\section{References}

1. Hansen, T.B. and A.D. Yaghjfan, Low-Frequency Scattering From Two-Dimensional Perfect Conductors, RADC Technical Report, 1991.

2. Jones, D.S., The Theory of Electromagnet1sm, MacMillan: New York, 1964, sec. 8.15.

3. Van Bladel, J., "Low-Frequency scattering by cylindrical bodies", Appl. Sc1. Res. Sec. B, 10, 195-202, 1963.

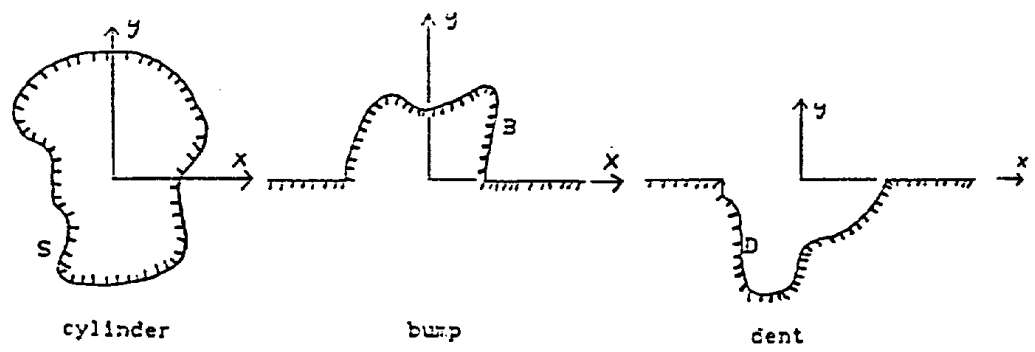

